\title{
Exact Uncertainty Principle and Quantization: Implications for the Gravitational Field
}

\author{
M. Reginatto \\ Physikalisch-Technische Bundesanstalt, Bundesallee 100, 38116 Braunschweig, Germany
}

Received on 23 December, 2004

\begin{abstract}
The quantization of the gravitational field is discussed within the exact uncertainty approach. The method may be described as a Hamilton-Jacobi quantization of gravity. It differs from previous approaches that take the classical Hamilton-Jacobi equation as their starting point in that it incorporates some new elements, in particular the use of a formalism of ensembles in configuration space and the postulate of an exact uncertainty relation. These provide the fundamental elements needed for the transition from the classical theory to the quantum theory.
\end{abstract}

\section{Introduction}

Perhaps one of the most fundamental distinctions between classical and quantum systems is that a quantum system must obey the uncertainty principle, while a classical system is not subject to such a limitation. The degree to which a quantum system is subject to this limitation is given by Heisenberg's uncertainty relation, which provides a bound on the minimum uncertainty of two conjugate variables such as the position $x$ and the momentum $p$ of a particle: it states that the product of their uncertainties must be equal to or exceed a fundamental limit proportional to Planck's constant, $\Delta x \Delta p \geq \hbar / 2$. This inequality, however, is not sufficiently restrictive to provide a means of going from classical mechanics to quantum mechanics. Indeed, in most approaches to quantization, no direct reference is made to the need for an uncertainty principle. For example, in the standard approach to canonical quantization, one introduces a Hilbert space, operators to represent observables, and a representation of the Poisson brackets of the fundamental conjugate variables in terms of commutators of their corresponding operators. Once this structure is in place, one can show that the Heisenberg uncertainty relations are indeed satisfied - but the uncertainty relations are derived, and the uncertainty principle, instead of motivating the method of quantization, seems to be little more than a consequence of the mathematical formalism.

One can, however, introduce an exact form of the uncertainty principle which is strong enough to provide a means of going from classical to quantum mechanics - indeed, this exact uncertainty principle provides the key element needed for such a transition. In particular, the assumption that a classical ensemble of particles is subject to nonclassical momentum fluctuations, of a strength inversely proportional to uncertainty in position, leads directly from the classical equations of motion to the Schrödinger equation $[1,2]$. This approach can also be generalized and used to derive bosonic field equations [3]. The exact uncertainty approach is extremely min- imalist in nature: unlike canonical quantization, no a priori assumptions regarding the existence of a Hilbert space structure, linear operators, wavefunctions, etc. is required. The sole "nonclassical" element needed is the addition of fluctuations to the momentum variable. The exact uncertainty approach is thus rather economical, in that it appears to make use of the minimum that is required for the description of a quantum system.

The paper is organized as follows. In the next two sections, I briefly review the case of a non-relativistic particle: in section 2, I discuss classical ensembles and derive the equations of motion from an ensemble Hamiltonian; in section 3 , I present the derivation of the Schrödinger equation using the exact uncertainty approach. In the remaining sections, I discuss the application of the exact uncertainty approach to gravity. A recent paper [4] provides an overview of the exact uncertainty approach in quantum mechanics and quantum gravity that emphasizes conceptual foundations. Therefore, in the last sections I focus instead on some technical issues and on some issues of interpretation. In section 4, I introduce the Hamilton-Jacobi formulation of general relativity and formulate a theory of classical ensembles of gravitational fields; in section 5, I apply the exact uncertainty principle to the gravitational field, which leads to a Wheeler-DeWitt equation. In section 6, I consider some implications of the exact uncertainty approach for the description of space-time in the quantized theory. Finally, some further remarks concerning the application of the exact uncertainty approach to gravity are given in section 7 .

\section{Classical ensembles of non- relativistic particles}

To motivate the introduction of classical ensembles in configuration space, consider the possibility that, due to either practical or theoretical reasons, the position of a particle is 
not known exactly. To describe this uncertainty in position, one has to introduce a probability density $P(x, t)$ on configuration space. The description of the physical system is then one that is given in terms of a statistical ensemble of particles. The dynamics of such an ensemble may be described using a Hamiltonian formalism, in the following way. For an ensemble of classical, non-relativistic particles of mass $m$ moving in a potential $V$, the correct equations of motion can be derived from the ensemble Hamiltonian

$$
\tilde{H}_{c}[P, S]=\int d x P\left(\frac{|\nabla S|^{2}}{2 m}+V\right) .
$$

The equations of motion that follow from eq. (1),

$$
\frac{\partial P}{\partial t}=\frac{\delta \tilde{H}_{c}}{\delta S}, \quad \frac{\partial S}{\partial t}=-\frac{\delta \tilde{H}_{c}}{\delta P}
$$

take the form

$$
\begin{gathered}
\frac{\partial P}{\partial t}+\nabla \cdot\left(P \frac{\nabla S}{m}\right)=0, \\
\frac{\partial S}{\partial t}+\frac{|\nabla S|^{2}}{2 m}+V=0 .
\end{gathered}
$$

Eq. (2) is a continuity equation for an ensemble of particles described by the velocity field $v=m^{-1} \nabla S$, and eq. (3) is the Hamilton-Jacobi equation for a classical particle with momentum $p=\nabla S$. Note also that $\tilde{H}_{C}$ is the average energy of the classical ensemble. The formalism of ensemble Hamiltonians that I have used here, while very simple, turns out to be quite general (see [5, 4] for more details on this formalism and various applications). In particular, it can be shown that both classical and quantum equations of motion can be derived using this formalism. The difference between classical and quantum ensembles can be characterized by a simple difference in the functional form of the corresponding ensemble Hamiltonians, as shown in the next section.

\section{Quantum equations for non- relativistic particles}

To derive quantum equations of motion, one needs to add fluctuations to the momentum variable which satisfy an exact uncertainty principle, and modify the classical ensemble Hamiltonian to take into consideration the effect of these fluctuations. There are, of course, further assumptions that need to be made, and these are explained in this section - however, the main conceptual ingredients are the nonclassical momentum fluctuations and the exact uncertainty principle.

Consider then another type of ensemble, one for which the assumption of a deterministic relation between position and momentum (implicit in the classical relation $p=\nabla S$ ) is no longer valid. Assume instead that the momentum is subject to stochastic perturbations, and that the relation between $p$ and $\nabla S$ takes the form

$$
p=\nabla S+f
$$

where the fluctuation field $f$ vanishes everywhere on average. Denoting the average over fluctuations by an overline, and using the relations $\bar{f}=0$ and $\bar{p}=\nabla S$, the classical ensemble energy is replaced in this case by

$$
\begin{aligned}
\langle E\rangle & =\int d x P\left(\frac{\overline{|\nabla S+f|^{2}}}{2 m}+V\right) \\
& =\tilde{H}_{c}+\int d x P \frac{\overline{f \cdot f}}{2 m}
\end{aligned}
$$

Thus the momentum fluctuations contribute to the average kinetic energy of this modified classical ensemble.

What conditions on the fluctuation field $f$ lead to the quantum equations of motion? It is sufficient to consider four principles $[1,2,3]$ (i) Action principle: The equations of motion can be derived from an action principle based on an ensemble Hamiltonian. Then, the strength of the fluctuations is determined by some function of $P$ and $S$ and their derivatives, i.e. $\overline{f \cdot f}=\alpha(x, P, S, \nabla P, \nabla S, \ldots)$. It will also be assumed that the equations of motion that result are partial differential equations of at most second order. (ii) Independence: if the system consists of two independent non-interacting sub-systems, the ensemble Hamiltonian, and therefore $\alpha$, decomposes into additive subsystem contributions. (iii) Invariance: the fluctuations transform correctly under linear canonical transformations (i.e., $f \rightarrow L^{T} f$ for any invertible linear coordinate transformation $x \rightarrow L x$. A sightly different principle was used in [1, 2]). (iv) Exact uncertainty principle: the strength of the momentum fluctuations is determined solely by the uncertainty in position. Since $P$ contains all the information on the position uncertainty, $\alpha$ can only depend on $P$ and its derivatives.

The first three principles are natural on physical grounds, and therefore relatively unconstraining. The fourth principle, however, by establishing a precise relationship between the position uncertainty (as described by $P$ ) and the strength of the momentum fluctuations, introduces a strongly nonclassical element. It is therefore appropriate to refer to the fluctuations due to the field $f$ as nonclassical momentum fluctuations. It can be shown that these four principles define the functional form of $\alpha$ uniquely and lead to a quantum ensemble Hamiltonian of the form

$$
\tilde{H}_{q}=\tilde{H}_{c}+C \int d x \frac{1}{P} \frac{|\nabla P|^{2}}{2 m}
$$

where $C$ is a positive universal constant. Furthermore, since the term $\alpha$ is proportional to the classical Fisher information, one can use the Cramér-Rao inequality of statistical estimation theory to derive an exact uncertainty relation that is stronger than (and implies) the Heisenberg uncertainty relation (see $[1,2,3]$ for derivations of these results).

It is straightforward to show that the Hamiltonian equations of motion for $P$ and $S$ derived from eq. (4),

$$
\frac{\partial P}{\partial t}=\frac{\delta \tilde{H}_{q}}{\delta S}, \quad \frac{\partial S}{\partial t}=-\frac{\delta \tilde{H}_{q}}{\delta P},
$$

are identical to the Schrödinger equation for a wavefunction $\psi$, provided one defines

$$
\hbar:=2 \sqrt{C}, \quad \psi:=\sqrt{P} e^{i S / \hbar} .
$$


As shown in [1], the wavefunction representations arises naturally from seeking canonical transformations which map the fields $P$ and $S$ to the "normal modes" of the system. This observation, together with a symmetry assumption, leads then to the usual Hilbert space formulation of quantum mechanics.

\section{Classical ensembles of gravitational fields}

The exact uncertainty approach can be succesfully generalized to derive the equations of motion for bosonic fields with Hamiltonians quadratic in the field momenta (e.g., scalar, electromagnetic and gravitational fields). The basic underlaying concept, the addition of nonclassical momentum fluctuations to a classical ensemble, carries through from particles to fields, although significant technical generalizations are needed (see [3] for details). In the rest of the paper, I discuss the application of the exact uncertainty approach to the gravitational field. However, before applying the exact uncertainty approach to the gravitational field, it will be necessary to introduce the Hamilton-Jacobi formulation of general relativity and to define a theory of classical ensembles consistent with this Hamilton-Jacobi formulation.

A Hamilton-Jacobi formulation for the gravitational field can be defined in terms of the functional equations

$$
\begin{gathered}
\mathcal{H}=\frac{1}{2} G_{i j k l} \frac{\delta S}{\delta h_{i j}} \frac{\delta S}{\delta h_{k l}}-\sqrt{h} R=0, \\
\mathcal{H}_{i}=-2 D_{j}\left(h_{i k} \frac{\delta S}{\delta h_{k j}}\right)=0 .
\end{gathered}
$$

where $R$ is the curvature scalar and $D_{j}$ the covariant derivative on a three-dimensional spatial hypersurface with (positive definite) metric $h_{k l}$, and

$$
G_{i j k l}=\frac{1}{\sqrt{h}}\left(h_{i k} h_{j l}+h_{i l} h_{j k}-h_{i j} h_{k l}\right) .
$$

is the DeWitt supermetric (units are chosen so that Newton's gravitational constant $G=1 / 16 \pi$ ) [6].

As a consequence of the Hamiltonian constraint $\mathcal{H}=0$, eq. (5), and the momentum constraints $\mathcal{H}_{i}=0$, eq. (6), $S$ must satisfy an infinite set of constraints, numbering four per three-dimensional point. $S$ also satisfies the condition $\frac{\partial S}{\partial t}=0$ [7]. The momentum constraints are equivalent to requiring the invariance of the Hamilton-Jacobi functional $S$ under spatial coordinate transformations. One may therefore formulate the theory in an equivalent way by keeping the Hamiltonian constraint, ignoring the momentum constraints, and requiring instead that $S$ be invariant under the gauge group of spatial coordinate transformations.

To define classical ensembles for gravitational fields, it is necessary to introduce some additional mathematical structure: a measure $D h$ over the space of metrics $h_{k l}$ and a probability functional $P\left[h_{k l}\right]$. A standard way of defining the measure $[8,9]$ is to introduce an invariant norm for metric fluctuations that depends on a parameter $\omega$,

$$
\|\delta h\|^{2}=\int d^{n} x[h(x)]^{\omega / 2} H^{i j k l}[h(x) ; \omega] \delta h_{i j} \delta h_{k l}
$$

where $n$ is the number of dimensions and

$$
H^{i j k l}=\frac{1}{2}[h(x)]^{(1-\omega) / 2}\left[h^{i k} h^{j l}+h^{i l} h^{j k}+\lambda h^{i j} h^{k l}\right]
$$

is a generalization of the inverse of the DeWitt supermetric (in [8] the particular case $\omega=0$ was considered). This norm induces a local measure for the functional integration given by

$$
\int d \mu[h]=\int \prod_{x}[\operatorname{det} H(h(x))]^{1 / 2} \prod_{i \geq j} d h_{i j}(x)
$$

where

$$
\operatorname{det} H(h(x)) \propto\left(1+\frac{1}{2} \lambda n\right)[h(x)]^{\sigma},
$$

and $\sigma=(n+1)[(1-\omega) n-4] / 4$ (one needs to impose the condition $\lambda \neq-2 / n$, otherwise the measure vanishes). Therefore, up to an irrelevant multiplicative constant, the measure takes the form

$$
\int d \mu[h]=\int \prod_{x}[\sqrt{h(x)}]^{\sigma} \prod_{i \geq j} d h_{i j}(x) .
$$

Without loss of generality, one may set $D h$ equal to $d \mu[h]$ with $\sigma=0$, since a term of the form $[\sqrt{h(x)}]^{\sigma}$ may be absorbed into the definition of $P\left[h_{k l}\right]$.

It is natural to require also that $\int D h P$ be invariant under the gauge group of spatial coordinate transformations. Since the family of measures defined by eq. (7) is invariant under spatial coordinate transformations $[9,10]$, the invariance of $\int D h P$ leads to a condition on $P$ that is similar to the one required of $S$. To show this, consider an infinitesimal change of coordinates $x^{\prime k}=x^{k}+\epsilon^{k}(x)$ and the corresponding transformation of the metric, $h_{k l} \rightarrow h_{k l}-\left(D_{k} \epsilon_{l}+D_{l} \epsilon_{k}\right)$. The variation of $\int D h P$ can be expressed as

$$
\delta_{\epsilon} \int D h P=\int D h\left[D_{k}\left(\frac{\delta P}{\delta h_{k l}}\right) \epsilon_{l}+D_{l}\left(\frac{\delta P}{\delta h_{k l}}\right) \epsilon_{k}\right] .
$$

Therefore, $\delta_{\epsilon} \int D h P=0$ requires

$$
D_{k}\left(\frac{\delta P}{\delta h_{k l}}\right)=0 \text {. }
$$

or the gauge invariance of $P$. In addition to eq. (8), it will be assumed that $\frac{\partial P}{\partial t}=0$ also holds.

Finally, it should be pointed out that one must factor out the infinite diffeomorphism gauge group volume out of the measure to calculate finite averages using the measure $D h$ and probability functional $P$. This can be achieved using the geometric approach described in [11]. This issue will not be discussed further here, since it does not affect the derivation of the equations of motion.

An appropriate ensemble Hamiltonian for the gravitational field is given by

$$
\tilde{H}_{c}=\sum_{x} \int D h P \mathcal{H} \sim \int d^{3} x \int D h P \mathcal{H} .
$$


The equations of motion derived from eq. (9) are of the form

$$
\frac{\partial P}{\partial t}=\frac{\Delta \tilde{H}_{c}}{\Delta S}, \quad \frac{\partial S}{\partial t}=-\frac{\Delta \tilde{H}_{c}}{\Delta P}
$$

where $\Delta / \Delta F$ denotes the variational derivative with respect to the functional $F$. With $\frac{\partial S}{\partial t}=\frac{\partial P}{\partial t}=0$, the equations of motion take the form

$$
\begin{gathered}
\mathcal{H}=0, \\
\int d^{3} x \frac{\delta}{\delta h_{i j}}\left(P G_{i j k l} \frac{\delta S}{\delta h_{k l}}\right)=0
\end{gathered}
$$

Eq. (10) is the Hamiltonian constraint, and eq. (11) may be interpreted as a continuity equation.

It is of interest that the interpretation of eq. (11) as a continuity equation leads to a rate equation that relates $\frac{\partial h_{k l}}{\partial t}$ and $\frac{\delta S}{\delta h_{k l}}$. This follows from the observation that such an interpretation is only possible if the "field velocity" $\frac{\partial h_{k l}}{\partial t}$ is related to $G_{i j k l} \frac{\delta S}{\delta h_{k l}}$ in a linear fashion. Indeed, the most general rate equation for the metric $h_{i j}$ that is consistent with the interpretation of eq. (11) as a continuity equation is of the form

$$
\delta h_{i j}=\left(\gamma G_{i j k l} \frac{\delta S}{\delta h_{k l}}+\delta_{\epsilon} h_{i j}\right) \delta t
$$

where $\gamma$ is an arbitrary function of $x$ (I have include a term $\delta_{\epsilon} h_{k l}=-\left(D_{k} \epsilon_{l}+D_{l} \epsilon_{k}\right)$ which allows for gauge transformations of $h_{k l}$, which is permitted because the gauge transformations are assumed to leave $\int D h P$ invariant, as discussed before). Therefore, one may write the rate equation for $h_{k l}$ in the standard form

$$
\frac{\partial h_{i j}}{\partial t}=N G_{i j k l} \frac{\delta S}{\delta h_{k l}}+D_{i} N_{j}+D_{j} N_{i} .
$$

Eq. (12) agrees with the equations derived from the ADM canonical formalism, provided $N$ is identified with the lapse function and $N_{k}$ with the shift vector [12].

\section{Quantum equations for gravita- tional fields}

The derivation of the quantum equations of motion from the exact uncertainty principle follows essentially the same steps as before [3]. It is first assumed that the field momenta are modified by a stochastic term, so that

$$
\pi^{k l}=\frac{\delta S}{\delta h_{k l}}+f^{k l}
$$

where $f^{k l}$ vanishes on average for all configurations, $\overline{f^{k l}}=$ 0 . The classical ensemble Hamiltonian $\tilde{H}_{c}$ is then replaced by a modified ensemble Hamiltonian $\tilde{H}_{q}$,

$$
\tilde{H}_{q}=\tilde{H}_{c}+\frac{1}{2} \int d^{3} x \int D h P G_{i j k l} \overline{f^{i j} f^{k l}}
$$

The form of the modified ensemble Hamiltonian is determined using the same principles as before (action principle, independence, invariance and exact uncertainty), leading to the result

$$
\tilde{H}_{q}=\tilde{H}_{c}+\frac{C}{2} \int d^{3} x \int D h \frac{1}{P} G_{i j k l} \frac{\delta P}{\delta h_{i j}} \frac{\delta P}{\delta h_{k l}}
$$

where $C$ is a positive universal constant. One can derive an exact uncertainty relation, defined in terms of generalized covariance matrices and Fisher information matrices, which connects the statistics of the metric field and its conjugate momentum fields and implies in turn a Heisenberg-type inequality (see [3] for derivations of these results).

If one now defines

$$
\hbar:=2 \sqrt{C}, \quad \Psi\left[h_{k l}\right]:=\sqrt{P} e^{i S / \hbar} .
$$

and makes use of the conditions $\frac{\partial S}{\partial t}=\frac{\partial P}{\partial t}=0$, it can be shown that the Hamiltonian equations that follow from eq. (13) lead to the Wheeler-DeWitt equation

$$
\left[-\frac{\hbar^{2}}{2} \frac{\delta}{\delta h_{i j}} G_{i j k l} \frac{\delta}{\delta h_{k l}}-\sqrt{h} R\right] \Psi=0 .
$$

Notice that the exact uncertainty approach specifies a particular operator ordering for the Wheeler-DeWitt equation. Furthermore, the Wheeler-DeWitt equation has been derived here using a quantization procedure based directly on the classical Hamilton-Jacobi formulation, instead of going through the usual canonical quantization procedure. Therefore, the difficult issues associated with the requirement of "Dirac consistency" (i.e., the need to find a choice of operator ordering and regularization scheme that will permit mapping the classical Poisson bracket algebra of constraints to an algebra of operators within the context of the Dirac quantization of canonical gravity) have been avoided. The only ambiguity that remains arises from the need to introduce some sort of regularization scheme to remove divergences arising from the product of two functional derivatives acting at the same point.

\section{Space-time}

Both the Hamilton-Jacobi theory of the classical gravitational field and the Wheeler-DeWitt equation involve functional equations defined on a three dimensional spatial hypersurface, with the group of spatial coordinate transformations as gauge group. The question then arises of whether it is possible to relate such formulations to field theories that give a description of space-time.

This is indeed the case for the classical theory, as is well known: one can show that the Hamilton-Jacobi formulation is equivalent to the usual formulation in space-time based on the Einstein equations $[13,6]$. The question becomes much more difficult when it comes to the quantized theory.

In section 4 , it was shown that the continuity equation derived from the classical ensemble Hamiltonian, eq. (11), implies the rate equation for the metric field, eq. (12). The same continuity equation and corresponding rate equation are derived from the quantum ensemble Hamiltonian, eq. (13). This has important implications in the quantum case, since it suggests a way of evolving away from the spatial surface 
using stochastic equations. Although the quantum ensemble Hamiltonian leads to a rate equation that is identical in form to the classical rate equation, the interpretation of this equation is somewhat different, because in the quantum case $\frac{\delta S}{\delta h_{k l}}$ represents an average over the non-classical fluctuations of the momentum. This suggests replacing eq. (12) by a stochastic rate equation

$$
\frac{\partial h_{i j}}{\partial t} \rightarrow N G_{i j k l}\left(\frac{\delta S}{\delta h_{k l}}+f^{k l}\right)+D_{i} N_{j}+D_{j} N_{i}
$$

and considering the time-evolved spatial metric as being subject to fluctuations. Furthermore, since the field momenta are subject to fluctuations, the extrinsic curvature tensor $K_{i j}$, defined by

$$
K_{i j}=\frac{1}{2} G_{i j k l} \frac{\delta S}{\delta h_{k l}}
$$

will also be subject to fluctuations, and should be replaced by a relation of the form

$$
K_{i j} \rightarrow \frac{1}{2} G_{i j k l}\left(\frac{\delta S}{\delta h_{k l}}+f^{k l}\right) .
$$

Since the extrinsic curvature tensor determines how spatial hypersurfaces fit together, it seems reasonable to expect the emergence of both well defined evolution equations and a well defined space-time limit after averaging over the nonclassical fluctuations, provided the fluctuations are not too violent.

\section{Discussion}

The approach to quantization of the gravitational field presented here may be described as a Hamilton-Jacobi quantization of gravity. It differs from previous approaches that take the classical Hamilton-Jacobi equation as their starting point in that it incorporates some new elements, in particular the use of the formalism of ensembles in configuration space and the postulate of the uncertainty relation as the fundamental element that is needed for the transition from the classical to the quantum theory. The additional mathematical structure required is minimal. No a priori assumptions regarding the existence of a Hilbert space structure, linear operators, wavefunctions, etc. or the use of path integrals are needed.

The use of ensemble Hamiltonians leads to a unified approach, one in which both classical and quantum equations are derived using the same mathematical formalism. In particular, it has been shown that the difference between classical and quantum ensembles is essentially a 'matter of form', being characterized by the additional term that is added to the classical ensemble Hamiltonian to quantize the system (i.e., eqs. (4) and (13)). This is of particular interest in the case of gravity, since a unified approach ensures that the quantum theory has a well defined classical limit. Furthermore, for any classical quantity that may be expressed as an average of a functional of $h_{k l}$ and $\frac{\delta S}{\delta h_{k l}}$, one may define a quantum counterpart by adding nonclassical fluctuations to the momentum; if this quantity has only linear and quadratic terms in $\frac{\delta S}{\delta h_{k l}}$, it will be possible to compute quantum corrections to the classical quantities in a straightforward way by averaging over the fluctuations. In this way, classical observables may be carried over into the quantum theory.

It is remarkable that already at the classical level, the theory of ensembles in configuration space provides new insight into general relativity. In particular, as pointed out in section 4 , the rate equation for the metric, eq. (12), appears as a direct consequence of the classical ensemble formalism of gravitational fields. In this way, it takes only a few steps to show that all 10 Einstein equations follow from the HamiltonJacobi formalism together with the assumption of a well defined ensemble of gravitational fields. On the other hand, the derivation from the Hamilton-Jacobi formalism alone [13], requires a much more lengthy derivation. The theory of classical ensembles in configuration space, which was introduced here as a starting point for the quantization of gravity, is interesting in its own right, and should be of useful for problems in which ensembles of gravitational fields need to be taken into consideration.

\section{Acknowledgment}

I am grateful to Michael J. W. Hall for his encouragement and many valuable discussions.

\section{References}

[1] M.J.W. Hall and M. Reginatto, J. Phys. A35 (2002) 3289

[2] M.J.W. Hall and M. Reginatto, Fortschr. Phys. 50 (2002) 646.

[3] M.J.W. Hall, K. Kumar and M. Reginatto, J. Phys. A36 (2003) 9779.

[4] M.J.W. Hall, in: Proceedings of the 4th Australasian Conference on General Relativity and Gravitation (Melbourne, January 7-9, 2004), to appear in GRG; preprint arXiv:grqc/0408098 v1

[5] M.J.W. Hall, J.Phys. A37 (2004) 7799.

[6] C.W. Misner, K.S. Thorne and J.A. Wheeler, Gravitation (Freeman, San Francisco, 1973).

[7] P.G. Bergmann, Phys. Rev. 144 (1966) 1078.

[8] B. DeWitt, in: General Relativity: An Einstein Centenary Survey, ed. by S.W. Hawking and W. Israel (Cambridge University Press, Cambridge, England, 1979).

[9] H.W. Hamber and R.M. Williams, Phys. Rev. D59 (1999) 064014

[10] L. Fadeev and V. Popov, Sov. Phys. Usp. 16 (1974) 777.

[11] E. Mottola, Jour. Math. Phys. 36 (1995) 2470.

[12] R. Wald, General Relativity (University of Chicago Press, Chicago, 1984).

[13] U.H. Gerlach, Phys. Rev. 177 (1969) 1929. 